\title{
Percutaneous computed tomography-guided biopsy performed by thoracic surgeons in 955 patients: A paradigm shift in image-guided thoracic procedures
}

Seth L. Welborn, MD, ${ }^{a}$ N. Paul Ohori, MD, ${ }^{b}$ Katie S. Nason, MD, MPH, ${ }^{a}$ Arjun Pennathur, MD, ${ }^{a}$

Omar Awais, DO, ${ }^{\mathrm{a}}$ Neil A. Christie, MD, ${ }^{\mathrm{a}}$ James D. Luketich, MD, ${ }^{\mathrm{a}}$ and Matthew J. Schuchert, MD ${ }^{\mathrm{a}}$

\section{ABSTRACT}

Objectives: Computed tomography-guided fine needle aspiration (CT-FNA) biopsy is a well-established diagnostic technique in the evaluation of lung nodules that is performed by radiologists in most centers. In this series, we analyzed the diagnostic and perioperative outcomes following CT-FNA performed by a dedicated group of thoracic surgeons.

Methods: We conducted a retrospective analysis of 955 patients undergoing CT-FNA by the thoracic surgery service. Primary outcome variables included diagnostic yield and accuracy, number of needle passes, complication rates, and adequacy of specimen for molecular testing.

Results: A satisfactory diagnostic specimen was obtained in $94.1 \%$ of cases. The average number of needle passes was $3.2 \pm 1.5$ (range, 1-10 passes). Diagnostic yield was significantly improved by increasing the number of passes from 1 to 2 to 3 passes $(P=.0003)$. CT-FNA diagnostic accuracy was $88.8 \%$. Diagnostic accuracy did not significantly improve with $\geq 4$ passes $(P=.20)$. Molecular testing was successful in $43.1 \%$, and did not improve with $\geq 4$ passes $(P=.5)$. Molecular testing success did improve with the addition of core needle biopsy $(P=.005)$. The pneumothorax rate for CT-FNA alone was $26.4 \%$, and increased with $\geq 4$ passes $(P=.009)$. The median length of stay for CT-FNA alone was 0 days (range, $0-74$ days), with same-day discharge in $67.5 \%$ of patients.

Conclusions: Thoracic surgeons can perform CT-FNA with excellent diagnostic yield and accuracy. Diagnostic yield, accuracy, and success in molecular testing do not improve with $\geq 4$ CT-FNA passes. Pneumothorax rates do increase with $\geq 4$ passes. The addition of core needle biopsy enhances success with molecular testing. (J Thorac Cardiovasc Surg 2019;157:1239-45)

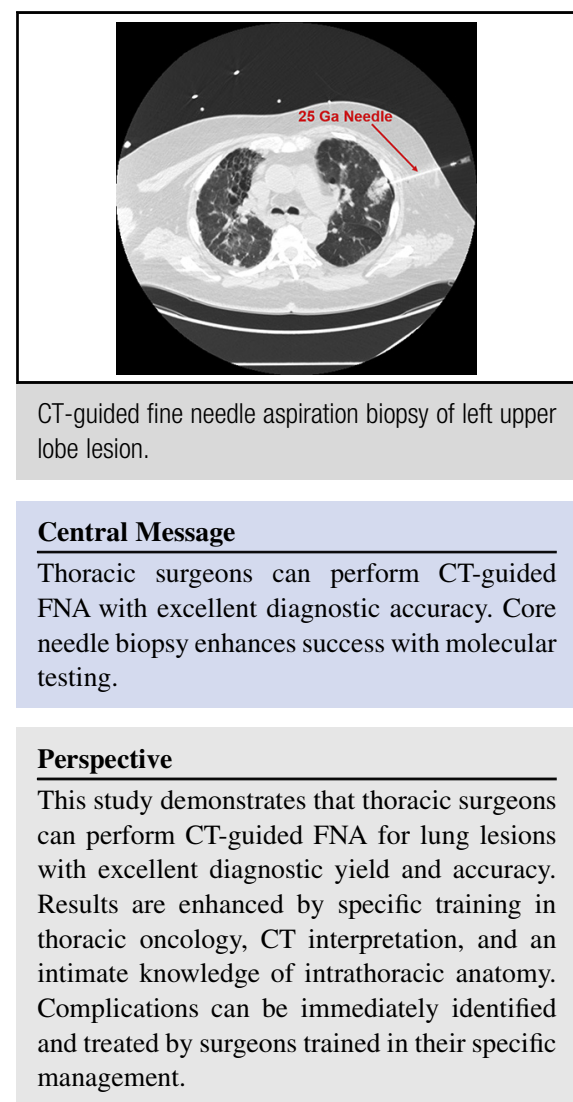

See Commentary on page 1246.

\footnotetext{
From the ${ }^{\mathrm{a}}$ Division of Thoracic and Foregut Surgery, Department of Cardiothoracic Surgery, and ${ }^{\mathrm{b}}$ Department of Pathology, University of Pittsburgh Medical Center, Pittsburgh, Pa.

Funding was provided by the Reid H. Chisholm Research Fund and the Department of Cardiothoracic Surgery.

Read at the 96th Annual Meeting of The American Association for Thoracic Surgery, Baltimore, Maryland, May 14-18, 2016.

Received for publication June 1, 2016; revisions received Aug 29, 2018; accepted for publication Sept 28, 2018; available ahead of print Dec 13, 2018.

Address for reprints: Matthew J. Schuchert, MD, Department of Cardiothoracic Surgery, 200 Lothrop St, Suite C-800, Pittsburgh, PA 15213 (E-mail: schuchertmj@upmc.edu).

$0022-5223 / \$ 36.00$

Copyright (c) 2018 by The American Association for Thoracic Surgery

https://doi.org/10.1016/j.jtcvs.2018.09.125
}

Computed tomography-guided fine needle aspiration (CT-FNA) biopsy is an important tool in the management of patients with pulmonary nodules and provides an accurate method of diagnosing malignancy. ${ }^{1}$ Traditionally, these procedures are performed by radiologists in imaging suites and are available in most centers nationwide. ${ }^{2}$ The

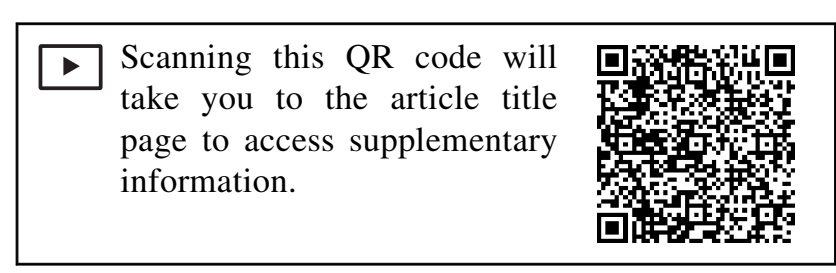




\section{Abbreviations and Acronyms \\ CT-FNA $=$ computed tomography fine needle aspiration \\ EMR $=$ electronic medical record \\ RFA = radiofrequency ablation \\ ROSE = rapid onsite evaluation \\ SBRT = stereotactic body radiation therapy}

detection of smaller and smaller lung nodules and advances in molecular testing have stimulated an enhanced need for accurate and expeditious tissue diagnosis, and have increased the demand for more tissue on a case-by-case basis. $^{3}$ The availability of CT imaging technology in modern hybrid operating rooms has introduced the possibility for thoracic surgeons to perform image-guided thoracic interventions during the evaluation and treatment of patients with lung nodules.

To date, there have been no published reports by thoracic surgeons regarding their experience with lung CT-FNA biopsy. At our center, thoracic surgeons have been actively involved in performing percutaneous procedures for more than 10 years. We reviewed our experience with CT-FNA biopsy in 955 patients. These procedures were primarily performed in a dedicated operating room with CT scanner and onsite cytopathologists to assist with intraoperative diagnosis. ${ }^{4}$ Primary end points evaluated included diagnostic yield, accuracy, and complication rates. Secondary end points included molecular testing success and the effect of number of passes on diagnostic parameters and complication rates (particularly pneumothorax).

\section{PATIENTS AND METHODS Patients}

Approval for this study was provided by the Institutional Review Board of the University of Pittsburgh (PRO14010256) and individual patient consent was waived. We performed a retrospective review of 955 patients undergoing CT-FNA biopsy of lung, mediastinal, or chest wall lesions by the thoracic surgery service at the University of Pittsburgh from May 2005 through March 2014. Patients were identified via billing records. Patients undergoing CT-FNA alone or in combination with other diagnostic or therapeutic procedures were included in this study. Patients were excluded for the following reasons: multiple nodules biopsied $(\mathrm{n}=55)$, extrathoracic sites $(n=19)$, no available electronic medical record (EMR) data/outside affiliate hospital $(\mathrm{n}=11)$, and concurrent navigational bronchoscopy performed $(n=9)$. Patient demographic characteristics and lung nodule characteristics are detailed in Table 1.

Patient selection for CT-FNA biopsy was individualized based on clinical and tumor characteristics. We will often request a CT-FNA biopsy to confirm malignancy before performing lobectomy for lesions that are otherwise not amenable to wedge resection based on lesion size, density, or location, especially in cases where the lesion appearance may be ambiguous based on $\mathrm{CT}$ or positron emission tomography characteristics or in higher-risk patients with a history of prior lung resection or those with marginal pulmonary function. A lobectomy for potentially benign disease in these situations is best avoided. CT-FNA biopsy is also used to confirm tissue diagnosis before performing higher-order resections
TABLE 1. Patient and lesion characteristics

\begin{tabular}{lc}
\hline \multicolumn{1}{c}{ Clinical variable } & Result \\
\hline Age (y) & $68.5 \pm 11.6$ \\
Gender (\%) & \\
$\quad$ Male & 49.2 \\
Female & 50.8 \\
Oxygen dependent (\%) & 7.7 \\
Prior history of cancer (\%) & 60.5 \\
Prior chest surgery (\%) & 22.3 \\
Tumor size (cm) & $3.0 \pm 2.1$ \\
Standardized uptake value & $7.5 \pm 6.2$ \\
Lesion location (\%) & \\
Pulmonary & 91.4 \\
Pleural & 3.1 \\
Chest wall & 3.0 \\
Mediastinal & 2.4 \\
\hline
\end{tabular}

Values for age, tumor size, and standardized update value are presented as mean \pm standard deviation.

(eg, sleeve resection, bilobectomy, or pneumonectomy), stereotactic body radiation therapy (SBRT)/stereotactic radiosurgery, or radiofrequency ablation (RFA).

Not all patients require CT-FNA biopsy. In some instances, for patients with acceptable risk, lobectomy is appropriate based on an overwhelming level of clinical suspicion, such that a negative CT-guided biopsy would not dissuade the decision to perform a resection. For peripheral lesions, wedge resection can be performed to confirm the tissue diagnosis with progression to lobectomy in the same setting, as indicated. Segmentectomy is also a useful approach that can be diagnostic and therapeutic in the setting of a solitary pulmonary nodule. ${ }^{5}$

\section{Surgical Technique}

Procedures were performed in a dedicated hybrid operating room with a built-in 64-slice CT scanner (GE Lightspeed VCT; General Electric Healthcare, Chicago, Ill). Patients are placed into either a supine or lateral position that permits access to all intrathoracic sites. A preprocedure CT scan is then performed. A typical protocol involves a noncontrast helical scan $(120 \mathrm{kV}, 120 \mathrm{~mA})$ with 2.5 -mm slices. The optimal skin entry site is established in reference to a fiducial skin marker. Biopsies are performed utilizing a $22 \mathrm{Ga}$ or $25 \mathrm{Ga} \times 9 \mathrm{~cm}$ needle (BD Spinal Needle; Becton, Dickinson and Co, Franklin Lakes, NJ). We prefer the $25 \mathrm{Ga}$ needle in most cases due to less blood contamination and higher specimen cellularity, as well as a lower pneumothorax potential. The position of the needle is tracked with limited image sets (either 9-slice helical set or 3-slice set utilizing the Smart Step CT-Fluoro mode). The specimen is then submitted to cytopathology for rapid onsite evaluation (ROSE).

Core biopsies were performed with a $20 \mathrm{Ga} \times 11 \mathrm{~cm}$ coaxial needle (Temno Evolution; Becton, Dickinson and Co) to acquire either 1 or $2 \mathrm{~cm}$ minicores. Multiple core samples were collected (typically 5-10 passes) to provide adequate tissue for ancillary testing. CT-guided percutaneous fiducial marker placement was performed utilizing preloaded 17 Ga needles (Cybermark Fiducial Markers; Civco Medical Solutions, Orange City, Iowa). Core biopsies are preferentially performed when there is a large mediastinal or pleural-based mass. For smaller intraparenchymal lesions, we perform CT-FNA to establish whether a lesion contains malignant cells. If the lesion is consistent with non-small cell carcinoma, we will proceed with core needle biopsies (if feasible) to collect adequate tissue for immunohistochemistry and molecular testing. RFA was performed with the Starburst Talon Semi-flex ablation probe (Angiodynamics, Latham, NY) as described previously. ${ }^{6}$ 


\section{Pathologic Evaluation}

ROSE was performed by dedicated cytopathologists in 873 cases $(91.4 \%)$. Cytologic specimens were graded as positive, suspicious, atypical, negative, or nondiagnostic in the final pathology report. Suspicious and atypical cytologic designations denote samples that are more or less likely to harbor malignancy, respectively, in cases where there is insufficient evidence on the slide to designate the result as clearly positive or negative.

Molecular testing denotes evaluating the tumor sample for specific genetic alterations, including such markers as epidermal growth factor receptor (mutation/deletion), Kirsten rat sarcoma viral gene homologue (mutation), tyrosine protein kinase MET (amplification), anaplastic lymphoma kinase (rearrangement), receptor tyrosine kinase rearranged during transfection (translocation), proto-oncogene tyrosine protein kinase ROS (rearrangement), proto-oncogene B-RAF (mutation), as well as programmed death-ligand 1 expression. Molecular testing success was defined as the percent of cases in which the pathologist had sufficient tissue to perform testing for the above genetic alterations.

\section{Data Analysis}

The 955 cases were stratified into 2 categories based on biopsy type for the analysis of diagnostic yield and accuracy. The group designated FNA includes cases in which tissue was sampled only through CT-FNA. The group labeled Core included all cases where core biopsy was utilized (the significant majority of these cases also had FNA performed as part of the procedure).

For analysis of complications, only pulmonary lesions were analyzed to not artificially reduce the overall complication rates. Three groups were chosen based on the extent of the percutaneous procedures performed. The group labeled FNA included only CT-FNA biopsy cases. The group labeled Core includes cases where core biopsy with or without CT-FNA biopsy was used. The group labeled Fiducial/RFA included cases where RFA and/or fiducial markers, with CT-FNA or core biopsy components, was performed.

\section{Diagnostic Parameters}

Diagnostic yield was defined as the percentage of biopsies with a tissue sample designated other than nondiagnostic. For the calculation of diagnostic accuracy, the postoperative sample labels were separated into positive and negative test results. For the purposes of analysis, cases designated as negative for malignancy, atypical, and nondiagnostic were classified as a clinically negative test result; suspicious and positive for malignancy were grouped as clinically positive test results. Final diagnosis was determined to be the diagnosis of the lesion at the time of definitive resection or definitive therapy (eg, SBRT). All lesions designated as suspicious on FNA that underwent RFA, SBRT/stereotactic radiosurgery, or were treated with chemotherapy or external beam radiation were considered clinically positive on final diagnosis.

\section{Follow-up}

Perioperative data were collected from the hospital chart, anesthesia, and operating room records as well as the EMR for each patient. Pneumothorax was defined as a pleural collection of air requiring placement of a pleural catheter (8.3 Fr pigtail catheter; Cook Lock Pericardiocentesis Set, Cook Medical, Inc, Bloomington, Ind). Perioperative mortality was defined as any death within 30 days or within the same hospital admission. Patients with negative biopsy results were evaluated longitudinally utilizing the EMR to determine whether the biopsied lesions were ever demonstrated to be malignant or were treated presumptively based on a high level of clinical suspicion.

\section{Statistical Analysis}

Both the Student $t$ test and the Wilcoxon test were used to compare the distributions of continuous data (eg, age, lesion size, chest tube duration, and length of stay), and the $\chi^{2}$ test or Fisher exact test was used to compare the frequencies of categorical measures (eg, gender, histology, and positive and negative test comparisons).

\section{RESULTS}

A total of 955 CT-FNA and/or CT-core needle biopsies were performed by 17 thoracic surgeons during the study period. The significant majority of procedures was performed by a core group, including authors MJS $(59.7 \%)$, NAC $(8.9 \%)$, OA $(5.4 \%)$, and $\mathrm{KN}(3.9 \%)$. Mean lesion size was $3.0 \pm 2.1 \mathrm{~cm}$. The biopsied intrathoracic lesions were distributed as follows: pulmonary $(91.5 \%)$, pleural $(3.1 \%)$, chest wall $(3.0 \%)$, and mediastinal $(2.4 \%)$. Final pathology revealed the following findings: 136 benign $(14.2 \%), 111$ atypical $(11.6 \%)$, 60 suspicious $(6.3 \%), 597$ positive $(62.5 \%)$, and 51 nondiagnostic $(5.3 \%)$.

\section{Diagnostic Parameters}

Overall diagnostic yield was $94.7 \%$ (95\% confidence interval $[\mathrm{CI}]$, 93.2-96.1). The diagnostic yield for FNA was $94.1 \%$ (95\% CI, 92.5-95.7), and improved to $98.3 \%$ $(95 \%$ CI, 96.1-99.9) with the use of core biopsy $(P=.052)$. Diagnostic sensitivity, specificity, and accuracy for FNA and core biopsies are detailed in Table 2. Disease prevalence in the study cohort was $77.2 \%$. Overall clinical sensitivity, specificity, and accuracy were $87.5 \%(95 \% \mathrm{CI}$, 85.1-89.9), 94.5\% (95\% CI, 91.5-97.5), and 89.1\% (95\% CI, 87.1-91.1), respectively. No significant difference in diagnostic accuracy was observed between FNA and Core groups. The size of the lesion had a significant effect on diagnostic yield. Overall, diagnostic yield increased from $88.2 \%$ to $96.5 \%(P=.0001)$ when sampling a lesion larger than $1.5 \mathrm{~cm}$, compared with lesions $<1.5 \mathrm{~cm}$. This was also seen in the FNA group $(87.8 \%$ vs $96.1 \% ; P=.0001)$. We found that ROSE was associated with a trend toward increased diagnostic accuracy (ROSE, $89.7 \%$ vs non-ROSE, $82.9 \% ; P=.065)$. Sensitivity and specificity of ROSE was $88.4 \%$ and $94.0 \%$, respectively.

\section{Number of FNA Passes}

The mean number of FNA passes was $3.2 \pm 1.5$ (median, 3 passes [range, 1-10 passes]). Use of ROSE increased number of needle passes from 2.81 to $3.23(P=.016)$.

Analysis of diagnostic yield, diagnostic accuracy, and molecular success rate revealed 3 distinct sets based on number of FNA passes (0-1, 2-3, and $\geq 4$ passes) (Table 3). Diagnostic yield improved significantly by utilizing 2 to 3 passes when compared with a single pass $(96.8 \%$ vs $86.5 \% ; P=.0003)$. However, improved diagnostic yield was not associated with 4 or more passes. In fact, it decreased significantly from $96.4 \%$ to $92.8 \%$ $(P=.012)$. A trend for an increase in diagnostic accuracy was observed with the use of 2 to 3 passes compared with a single pass $(P=.063)$. 
TABLE 2. Diagnostic parameters by biopsy type

\begin{tabular}{lccc}
\hline & & \multicolumn{2}{c}{ Type of biopsy } \\
\cline { 3 - 4 } Diagnostic parameter & Overall & Fine needle aspiration & Core \\
\hline True positive & 645 & 565 & 80 \\
True negative & 206 & 176 & 30 \\
False positive & 12 & 10 & 2 \\
False negative & 92 & 83 & 9 \\
\hline Sensitivity & $87.5(85.1-89.9)$ & $87.2(84.6-89.8)$ & $89.9(83.6-96.2)$ \\
Specificity & $94.5(91.5-97.5)$ & $94.6(91.4-97.9)$ & $93.8(85.4-100.0)$ \\
Diagnostic accuracy & $89.1(87.1-91.1)$ & $88.8(86.7-91.0)$ & $90.9(85.8-96.0)$ \\
Positive predictive value & $98.2(97.2-99.2)$ & $98.3(97.2-99.3)$ & $97.6(94.2-100.0)$ \\
Negative predictive value & $69.1(63.9-74.4)$ & $68.0(62.3-73.6)$ & $76.9(63.7-90.1)$ \\
\hline Val
\end{tabular}

Values are presented as $\mathrm{n}$ or $\%(95 \%$ confidence interval).

\section{Molecular Testing}

Molecular testing was attempted in 157 biopsies $(16.4 \%)$, and was successful in 76 cases $(48.4 \%)$. Molecular success for FNA was $43.1 \%$, and improved significantly to $74.1 \%$ with the addition of core biopsies $(P=.005)$. Success in molecular testing also correlated with increasing tumor size. Success in molecular testing was seen in $34.0 \%$ of patients with tumors $\leq 2 \mathrm{~cm}$, and increased significantly with tumors $>2 \mathrm{~cm} \quad(54.5 \%$; $P=.023)$. Molecular testing success did not correlate with an increased number of FNA passes (Table 3).

\section{Perioperative Course and Complications}

The vast majority of cases $(91.5 \%)$ were performed as planned outpatient procedures. The median overall length of stay for outpatient cases was 0 days (mean, $1.0 \pm 4.3$ days [range, 0-93 days]). Same-day discharge was accomplished in $64.1 \%$ of patients. Length of stay was significantly increased for the RFA/Fiducial group compared with the FNA group $(P=.0001)$ (Table 4). Median overall chest tube duration was 1 day (mean, $2.6 \pm 5.8$ days [range, 0-70 days]). There was no significant difference in chest tube duration between any of the groups. Median overall operative time was 45 minutes (mean, $50 \pm 17$ minutes [range, 15-123 minutes]). Mean operative time was significantly longer for the RFA/Fiducial group compared with the FNA group $(P=.0001)$.
The primary complication analyzed was pneumothorax rate: $29.7 \%$ overall $(95 \% \mathrm{CI}, 26.8 \%-32.6 \%)$. The vast majority of pneumothoraxes are detected in the operating room, with $<5 \%$ of cases developing in a delayed fashion in the postanesthesia care unit (1-2 hours following the procedure) or during inpatient observation. Factors that affected pneumothorax rate included number of CT-FNA passes and lesion size. Pneumothorax rates in the FNA group increased when using 4 or more passes $(32.6 \%$ vs $22.3 \% ; P=.0047$ ), and when sampling a lesion $<1.0 \mathrm{~cm}$ $(45.5 \%$ vs $26.2 \%$ for lesions $\geq 1 \mathrm{~cm} ; P=.015)$. Pneumothorax rates were significantly lower for the Core group $(P=.021)$, and higher for the RFA/Fiducial group $(P=.0001)$ with respect to the FNA group (Table 4).

Secondary complications included pleural effusion requiring drainage $(2.0 \%)$, intensive care unit admission as a result of the procedure $(1.9 \%)$, hemoptysis $(0.9 \%)$, and infection $(0.5 \%)$. All-cause perioperative mortality was $0.7 \%$; procedure-related mortality was $0.5 \%$ (sequelae of pre- or postoperative pulmonary embolism in 3 cases, stroke in 1 case, and preoperative pneumonia that worsened postoperatively in 1 case). The remaining 2 cases expired after lobectomy and an unrelated vascular surgery procedure within 30 days.

\section{Procedure Trends}

The mean number of passes increased from $2.3 \pm 1.3$ in 2005 to $4.9 \pm 2.1$ in $2014(P=.0009)$, and the percent of

TABLE 3. Diagnostic yield, accuracy, and molecular testing success rates stratified by number of computed tomography fine needle aspiration passes

\begin{tabular}{|c|c|c|c|c|c|c|c|c|c|}
\hline \multirow[b]{3}{*}{ Parameter } & \multicolumn{9}{|c|}{ Number of passes } \\
\hline & \multicolumn{3}{|c|}{1} & \multicolumn{3}{|c|}{2 to 3} & \multicolumn{3}{|c|}{$\geq 4$} \\
\hline & $\%$ & $\bar{n}$ & $95 \% \mathrm{CI}$ & $\%$ & n & $95 \% \mathrm{CI}$ & $\%$ & n & $95 \% \mathrm{CI}$ \\
\hline Diagnostic yield & 86.5 & 89 & $79.4-93.6$ & 96.8 & $439 *$ & $95.2-98.5$ & 92.8 & 306 & $89.9-95.7$ \\
\hline Diagnostic accuracy & 83.1 & 89 & $75.4-90.9$ & 90.2 & 439 & $87.4-93.0$ & 88.6 & 306 & $85.0-92.1$ \\
\hline Molecular success rate & 57.1 & 7 & $20.5-93.8$ & 44.0 & 50 & $30.2-57.8$ & 41.1 & 73 & $29.8-52.4$ \\
\hline
\end{tabular}

$C I$, Confidence interval. $* P<.05$ compared with single pass. 
TABLE 4. Perioperative outcomes by procedure type

\begin{tabular}{lcccc}
\hline & & \multicolumn{2}{c}{ Procedure } \\
\cline { 2 - 5 } \multicolumn{1}{c}{ Outcome } & Overall & Fine needle aspiration & Core & Radiofrequency ablation/fiducial \\
\hline Operative time (min) & $50 \pm 17.1(45[15-123])$ & $49 \pm 15(45[15-120])$ & $50 \pm 23(45[20-120])$ & $68 \pm 25^{*}(65[26-123])$ \\
Outpatient length of stay (d) & $1.0 \pm 4.3(0[0-94])$ & $0.8 \pm 4.1(0[0-94])$ & $0.5 \pm 1.0(0[0-7])$ & $2.4 \pm 6.3^{*}(1[0-56])$ \\
Chest tube duration (d) & $2.6 \pm 5.8(1[0-70])$ & $2.5 \pm 5.6(1[0-70])$ & $1.8 \pm 1.6(1[1-7])$ & $3.1 \pm 6.6(1[0-56])$ \\
Pneumothorax $(\%)$ & 29.7 & 26.4 & $14.5^{*}$ & $53.5^{*}$ \\
\hline
\end{tabular}

Values are presented as mean \pm standard deviation (median [range]) unless otherwise noted. $* P<.05$ compared with fine needle aspiration.

cases with a core needle biopsy component increased from $11.1 \%$ before 2013 to $22.5 \%$ after 2013 ( $P=.0009$ ). Combining the FNA and Core groups together, the pneumothorax rate increased from $22.5 \%$ before 2013 , to $40.8 \%$ during 2013-2014 $(P=.0002)$. The relationship between number of FNA passes, use of core needle biopsy and pneumothorax rate over time is shown in Figure 1. The rate of attempted molecular testing increased from $3.5 \pm 3.6$ cases/year before 2011 to $34.0 \pm 26.2$ cases/year from 2011-2014 $(P=.0001)$.

\section{DISCUSSION}

CT-FNA biopsy remains the modality of choice in the diagnosis of pulmonary nodules. ${ }^{7}$ Diagnostic yield and accuracy rates vary from $65 \%$ to $96 \%$ in published studies. ${ }^{8}$ Current joint guidelines from the American College of Radiology and the Society of Interventional Radiology recommend a diagnostic yield of at least $75 \%,{ }^{9}$ typically requiring 1 to 3 passes to achieve this threshold. The diagnostic yield in the current study $(94.7 \%)$ compares favorably to this standard. The diagnostic accuracy of $89.1 \%$ of CT-FNA procedures with or without core needle biopsy is also higher than most reported series. ${ }^{10}$ Several factors may be contributing to the enhanced accuracy observed in this analysis. The significant majority of patients $(>98 \%)$ have been evaluated by thoracic surgeons in outpatient settings before the procedure, which will serve to identify lesions with a higher probability of malignancy. Another factor that has been shown to play an important role in enhancing diagnostic accuracy is ROSE. ${ }^{11}$ Sharp reductions in nondiagnostic/inadequate specimens and of false negatives have been reported with intraoperative assessment. ${ }^{4}$ We found a similar trend toward increased diagnostic accuracy with ROSE (ROSE, $89.7 \%$ vs non-ROSE: $82.9 \% ; P=.065$ ).

Pneumothorax represents the most common complication of CT-FNA/core needle biopsies, ranging from $9 \%$ to $54 \% .^{12}$ Pneumothorax requiring insertion of a pleural catheter generally is observed in $<20 \%$ of reported series. ${ }^{9}$ Although our overall pneumothorax rate is within reported norms, our pneumothorax rate with associated pleural catheter insertion of $26.4 \%$ following CT-FNA biopsy is higher than that reported in most radiology series. There are likely several reasons for this. First, we have a low threshold for placing a pigtail catheter when a moderate-large pneumothorax is encountered. This more aggressive therapeutic posture is intended to minimize the risk of delayed pneumothorax that could necessitate the placement of pleural catheters in the recovery room or on the hospital ward, or result in readmission to a hospital after discharge. In contradistinction, most radiologists without admitting privileges will be more likely to attempt conservative management through observation or aspiration. Pneumothorax rates have been shown to correlate with tumor size, ${ }^{13}$ number of needle passes performed,${ }^{14}$ needle size, ${ }^{15}$ and chronic obstructive pulmonary disease severity. ${ }^{16}$ We similarly found that that pneumothorax rates increased significantly for lesions $<1 \mathrm{~cm}$ in size, and when 4 or more passes were performed. A more selective approach at pleural catheter insertion by

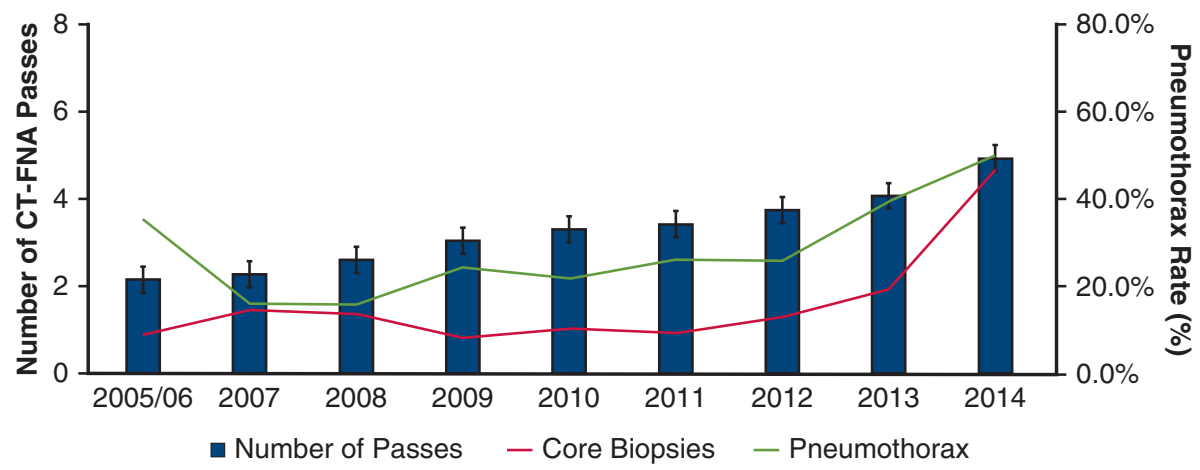

FIGURE 1. Increasing number of computed tomography fine needle aspiration (CT-FNA) passes, core needle biopsy use, and pneumothorax rates over time. 
attempting an aspiration at the end of the case might reduce the overall requirement for pleural catheter insertion and admission, but could lead to an increase in the number of catheters placed in the recovery room or perhaps even after discharge. Navigational bronchoscopy has emerged as an attractive alternative to CT-FNA that is associated with a reduced risk of pneumothorax. We will consider this procedure in the setting of tumors confined to the inneror middle-third of the lung with a bronchus sign. ${ }^{17}$

In the era of individualized cancer care, we have witnessed a significant increase in the demand for tissue both for immunohistochemistry and molecular testing. Correspondingly, we witnessed an increase in the number of passes performed in an effort to acquire more tissue (Figure 1). Despite this effort, yield in molecular testing has not improved with increased CT-FNA passes. Furthermore, we have observed an increase in the pneumothorax rate with an increased number of passes (Figure 1). In our experience, CT-FNA alone was sufficient for molecular testing in only $48.4 \%$ of cases. Tumor size did seem to correlate with molecular success (Figure 2). The addition of core needle biopsy was associated with a significant improvement in molecular testing success, and did not appear to augment the pneumothorax rate. A potential reason why core needle biopsies were associated with a lower pneumothorax rate in this study is that we generally perform core biopsies in larger, more peripheral lesions where the needle does not need to traverse large areas of aerated lung. Another factor is that the coaxial core biopsy system can permit access to the target lesion using a 19 Ga guide needle, allowing the ability to obtain multiple $20 \mathrm{Ga}$ cores through a single pleural puncture. We, therefore, recommend performing core needle biopsies early in suitable lesions where molecular testing is warranted.

What is the role of thoracic surgeons in image-guided interventions? Thoracic surgeons have intensive training in the principles of thoracic oncology, in thoracic image interpretation, and have an intimate 3-dimensional understanding of intrathoracic anatomy. By being involved in the outpatient workup, surgeons are uniquely equipped to assess the clinical index of suspicion and weigh the risk-to-benefit ratio before embarking on a given procedure. Surgeons are specifically trained to perform the technical aspects of these procedures and have the capability to expeditiously identify and treat any procedure-related complication in operating room settings. Unlike radiologists in most centers, surgeons have admitting privileges and follow their patients routinely, which enhances outcomes and quality assurance. Furthermore, therapeutic interventions (eg, RFA and fiducials) can be performed immediately upon securing a diagnosis, even in the same setting as CT-FNA. For thoracic surgery credentialing, we recommend that practitioners have

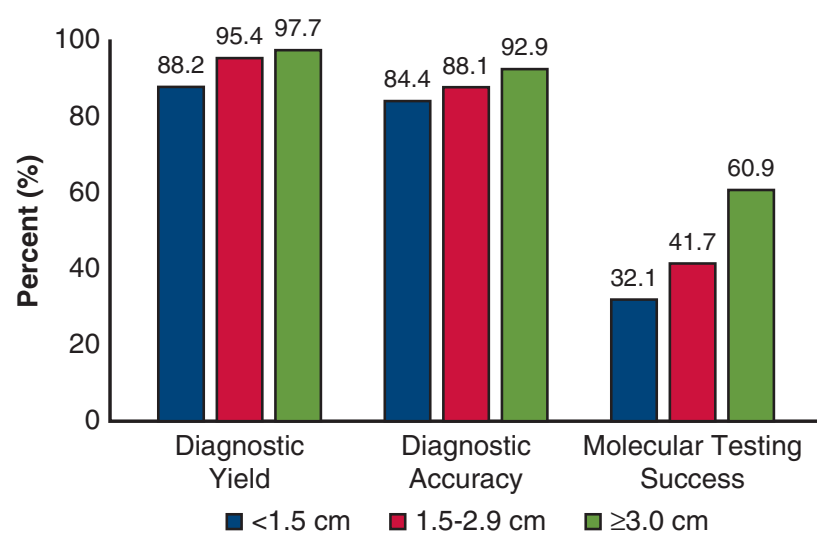

FIGURE 2. Diagnostic yield, accuracy, and molecular testing success stratified by tumor size.

completed a thoracic surgery residency program, obtain formal didactic education for CT- and ultrasound-guided interventions, and have performed 5 proctored CT-FNA procedures (Video 1).

There are many limitations to this study. The retrospective nature of this study makes unplanned comparisons between groups subject to both surgeon and selection bias. Surgical approaches have evolved during the study period (eg, demand for more tissue, increased FNA passes, and more liberal use of core biopsy), which makes evaluating the outcomes over differing time periods inherently more difficult. Hybrid operating rooms are not available in all centers, which can present a logistic barrier to performing these procedures. We have found that sharing the operating room time in a multidisciplinary fashion with allied surgical specialties (eg, neurosurgery) optimizes block use and enhances negotiations with administration to build a hybrid operating room. Lastly, this study does

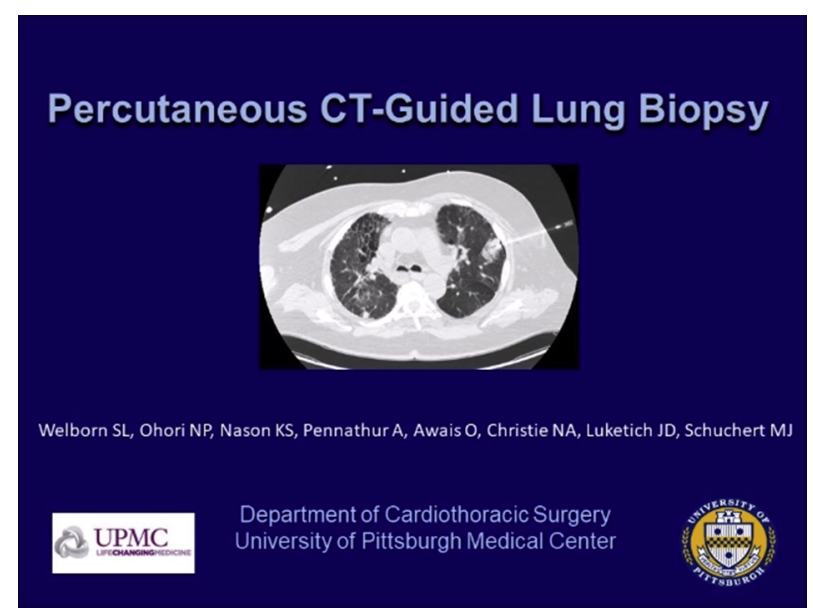

VIDEO 1. Percutaneous computed tomography-guided lung biopsy. Video available at: https://www.jtcvs.org/article/S0022-5223(18)32939-8/ fulltext. 
not take into account any resource management or cost analysis. These factors are of considerable importance, and should be considered when deciding whether or not these procedures can/should be added to a surgeon's scope of practice.

\section{CONCLUSIONS}

Thoracic surgeons can perform CT-FNA biopsy with excellent diagnostic yield and accuracy. Biopsies can be performed on lesions $<1.5 \mathrm{~cm}$ in size while maintaining diagnostic yield rates consistent with interventional radiology guidelines. Only when the lesion size is $<1.0 \mathrm{~cm}$ is an increased pneumothorax rate observed. Diagnostic yield, accuracy, and success in molecular testing do not appear to improve with $\geq 4$ FNA passes. Pneumothorax rates, however, do increase with $\geq 4$ passes. The addition of core needle biopsy does enhance success with molecular testing. Presently, we perform 2 to 3 CT-FNA passes for diagnosis, and switch to core needle biopsy to obtain tissue for ancillary studies. When obtaining tissue for ancillary studies, a more liberal use of core needle biopsy, as opposed to increased CT-FNA passes, appears warranted.

\section{Conflict of Interest Statement}

Authors have nothing to disclose with regard to commercial support.

\section{References}

1. Silverman JF, Finley JL, O'Brien KF, Dabbs DJ, Park HK, Larkin EW, et al. Diagnostic accuracy and role of immediate interpretation of fine needle aspiration biopsy specimens from various sites. Acta Cytol. 1989;33:791-6.

2. Khan KA, Zaidi S, Swan N, Browne R, Torreggiani W, Lane S, et al. The use of computerized tomography guided percutaneous fine needle aspiration in the evaluation of solitary pulmonary nodules. Ir Med J. 2012;105:50-2.

3. Ng YL, Patsios D, Roberts H, Walsham A, Paul NS, Chung T, et al. CT-guided percutaneous fine-needle aspiration biopsy of pulmonary nodules measuring $10 \mathrm{~mm}$ or less. Clin Radiol. 2008;63:272-7.
4. Mazza E, Maddau C, Ricciardi A, Falchini M, Matucci M, Ciarpallini T. On-site evaluation of percutaneous CT-guided fine needle aspiration of pulmonary lesions. A study of 321 cases. Radiol Med. 2005;110:141-8.

5. Schuchert MJ, Abbas G, Awais O, Pennathur A, Nason KS, Wilson DO, et al Anatomic segmentectomy for the solitary pulmonary nodule and early-stage lung cancer. Ann Thorac Surg. 2012;93:1780-7.

6. Pennathur A, Abbas G, Gooding WE, Schuchert MJ, Gilbert S, Christie NA, et al. Image-guided radiofrequency ablation of lung neoplasm in 100 consecutive patients by a thoracic surgical service. Ann Thorac Surg. 2009; 88:1601-8.

7. Hasanovic A, Rekhtman N, Sigel CS, Moreira AL. Advances in fine needle aspiration cytology for the diagnosis of pulmonary carcinoma. Patholog Res Int. 2011;2011:1-7.

8. Kothary N, Lock L, Sze DY, Hoffman LV. Computed tomography-guided percutaneous needle biopsy of pulmonary nodules: impact of nodule size on diagnostic accuracy. Clin Lung Cancer. 2009;10:360-3.

9. Gupta S, Walalce MJ, Cardella JF, Kundu S, Miller DL, Rose SC. Quality improvement guidelines for percutaneous needle biopsy. J Vasc Intern Radiol. 2010;21:969-75.

10. Nizzoli R, Tiseo M, Gelsomino F, Bartolotti M, Majori M, Ferrari L, et al Accuracy of fine needle aspiration cytology in the pathological typing of non-small cell lung cancer. J Thorac Oncol. 2011;6:481-93.

11. Austin JHM, Cohen MB. Value of having a cytopathologist present during percutaneous fine-needle aspiration biopsy of lung: report of 55 cancer patients and meta-analysis of the literature. AJR Am J Roentgenol. 1993;160: $175-7$.

12. Boskovic T, Stanic J, Pena-Karan S, Zarogoulidis P, Drevelegas K, Katsikogiannis N, et al. Pneumothorax after transthoracic needle biopsy of lung lesions under CT guidance. J Thorac Dis. 2014;6(Suppl 1):S99-107.

13. Cox JE, Chiles C, McManus CM, Acquino SL, Choplin RH. Transthoracic needle aspiration biopsy: variables that affect risk of pneumothorax. Radiology. 1999;212:165-8.

14. Kakizawa H, Toyota N, Hieda M, Tachikake T, Matsuura N, Oda M, et al. Risk factors for severity of pneumothorax after CT-guided percutaneous lung biopsy using the single-needle method. Hiroshima J Med Sci. 2010;59:43-50.

15. Geraghty PR, Kee ST, McFarlane G, Razavi MK, Sze DY, Dake MD. CT-guided transthoracic needle aspiration biopsy of pulmonary nodules: needle size and pneumothorax rate. Radiology. 2003;229:475-81.

16. Fish GD, Stanley JH, Miller KS, Schabel SI, Sutherland SE. Postbiopsy pneumothorax: estimating the risk by chest radiography and pulmonary function tests. AJR Am J Roentgenol. 1988;150:71-4.

17. Awais O, Reidy MR, Mehta K, Bianco V, Gooding WE, Schuchert MJ, et al Electromagnetic navigation bronchoscopy-guided dye marking for thoracoscopic resection of pulmonary nodules. Ann Thorac Surg. 2016;102:223-9.

Key Words: biopsy, fine needle aspiration, diagnostic yield, diagnostic accuracy, core biopsy, pneumothorax 